\title{
BMJ Open An investigation of general predictors for cognitive-behavioural therapy outcome for anxiety disorders in a routine clinical setting
}

\author{
Sara Kerstine Kaya Nielsen, ${ }^{1}$ Signe Vangkilde, ${ }^{1}$ Kate B Wolitzky-Taylor, ${ }^{2}$ \\ Sarah Ingrid Franksdatter Daniel, ${ }^{1}$ Ida Hageman ${ }^{3}$
}

To cite: Nielsen SKK, Vangkilde S, WolitzkyTaylor KB, et al. An investigation of general predictors for cognitivebehavioural therapy outcome for anxiety disorders in a routine clinical setting. BMJ Open 2016;6:e010898. doi:10.1136/bmjopen-2015010898

- Prepublication history for this paper is available online. To view these files please visit the journal online (http://dx.doi.org/10.1136/ bmjopen-2015-010898).

Received 17 December 2015 Revised 16 February 2016 Accepted 7 March 2016

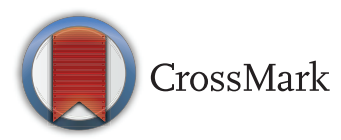

${ }^{1}$ Department of Psychology, University of Copenhagen, Copenhagen, Denmark ${ }^{2}$ Department of Psychiatry and Biobehavioral Sciences Semel Institute, University of California, California, USA

${ }^{3}$ Psychiatric Centre

Copenhagen, Copenhagen University Hospital, Copenhagen, Denmark

Correspondence to Sara Kerstine Kaya Nielsen; Sara.nielsen@psy.ku.dk

\begin{abstract}
Introduction: Cognitive-behavioural therapy (CBT) is effective for treating anxiety disorders and is offered in most mental health services around the world.

However, a relatively large number of patients with anxiety disorders do not benefit from CBT, experience relapses or drop out. Reliable predictors of treatment effects are lacking. The aim of this study is to investigate the predictive value of emotion regulation and attentional control for CBT outcome in a routine setting.
\end{abstract}

Methods and analysis: In this prospective and practice-based study, 112 patients with anxiety disorders referred for manual-based group CBT at two psychiatric outpatient clinics will be recruited. Emotion regulation, severity of anxiety and attentional control will be assessed with self-report measures and with an experimental computer-based attentional control task at baseline, post-treatment and at a 6-month follow-up.

Emotion regulation will be measured with Difficulties in Emotion Regulation Questionnaire, severity of anxiety will be assessed with Beck Anxiety Inventory and attentional control will be measured with the self-report questionnaire, Attention Control Scale, and with an experimental computer-based attentional control task based on theory of visual attention. Data will be analysed using multilevel mixed-effects modelling. Ethics and dissemination: The study is approved by the Danish National Ethical Board, the Department of Psychology Ethical Board, University of Copenhagen and by the Danish Data Protection Agency. Study findings will be disseminated through peer-reviewed journal publications and conference presentations. The Danish Committee System on Health Research Ethics has been notified about the project.

Trial registration number: NCT02638363.

\section{INTRODUCTION}

Anxiety disorders are a pervasive problem, with an estimated 12-month prevalence of $16.6 \%,{ }^{1}$ and they are associated with significant impairment ${ }^{2}$ and high social costs. ${ }^{3}$

\section{Strengths and limitations of this study}

- One of the only practice-based predictor studies.

- First study to examine the predictive value of attentional control.

- The study combines experimental and self-report measures.

- The study does not include an untreated control arm.

Cognitive-behavioural therapy (CBT) has shown to be effective in treating anxiety disorders ${ }^{4}$ for meta-analytic review. CBT is furthermore cost-effective. ${ }^{5}$ This has made CBT the first choice of treatment for anxiety disorders in many mental health settings around the world. Despite this impressive evidence base, a relatively large group of individuals do not benefit from CBT, relapse or drop out of treatment. ${ }^{6}$ Thus, there is a clear and pressing need to improve the efficacy and effectiveness of CBT for anxiety disorders. One way to improve is to identify predictors of CBT outcome. In routine clinical settings, identification of prognostic factors can help allocate resources to those presumed to benefit most from CBT, while also identifying those who may need additional CBT, adjunctive therapy or entirely different treatment to maximise outcomes.

Current knowledge about who is most likely to benefit from CBT is sparse and stems primarily from well-controlled laboratory settings. These results may not generalise to patient outcomes in mental health settings in which patients with more severe and comorbid disorders are often seen. ${ }^{7-10}$ Thus, practice-based studies (ie, studies conducted in routine clinical settings) have the potential to significantly increase knowledge, inform clinical strategies, and thus improve the treatment offered in mental health 
settings. ${ }^{10}$ Furthermore, existing studies have mostly investigated sociodemographic factors as predictors of drop out and non-response. However, these variables have shown to be either non-significant or inconsistent predictors of outcome. ${ }^{11}$ Results concerning comorbidity as a predictor are also mixed. ${ }^{12}{ }^{13}$ In sum, knowledge on who is most likely to benefit from CBT is sparse, and to date no reliable predictors have been identified. ${ }^{14} 15$

While prior work has primarily emphasised atheoretical predictors, it may prove more fruitful to focus on predictor variables stemming from psychological theories. Identifying such predictors can provide direct prognostic information for clinicians. Additionally, these predictors could also inform the development of adjunctive components of treatment that may optimise the treatment for those with poorer CBT outcome. Some exciting studies suggest that such a line of inquiry could yield relevant results. ${ }^{16} 17$

Maintaining factors is one aspect highlighted by several therapeutic approaches/theories as crucial for the treatment of anxiety disorders, and accordingly these factors are often the target of cognitive and behavioural treatments. Emotion regulation and attentional control are examples of key maintaining factors in anxiety disorders. ${ }^{18-23}$

CBT, which aims to teach patients cognitive reappraisal of maladaptive cognitions and encourages approach (rather than avoidance) behaviour via cognitive restructuring and exposure, ${ }^{24}$ may be more suitable for patients with certain characteristics. Specifically, recent research and theory suggest that it is crucial for effective treatment of anxiety disorders that clients learn to tolerate fear while being exposed to anxiety-provoking stimuli, rather than merely habituating to the stimuli. ${ }^{25}$ Learning to tolerate fear may require emotion regulation skills and the ability to control attention and this has been supported by recent studies. ${ }^{25}$ However, so far only certain areas of emotion regulation have been investigated and to date never in a routine clinical setting. Attentional control has never been examined as a predictor of CBT outcomes. Accordingly, this study is designed to investigate the predictive value of attentional control and of three facets of emotion regulation skills.

\section{Attentional control}

There is strong evidence that individuals with anxiety disorders have biased attention towards threatening stimuli, ${ }^{18}$ but there is no consensus as to why these biases occur. Recent research suggest that it is not the threat bias per se that is problematic. Rather, individual differences in the ability to voluntarily control attention may affect the impact of threatening information, hence increasing or decreasing the anxiety symptoms. ${ }^{20}{ }^{21} \mathrm{~A}$ potential consequence may be that individuals with low ability to voluntarily control their attention have difficulties participating in exposure and/or do not progress in the exposure hierarchy, because the level of anxiety gets too high. This may subsequently lead to failure to learn that fear is not dangerous or even drop out of CBT. Furthermore, a pronounced bias towards irrelevant negative information may take up so many resources that processing of important, relevant information is hampered, making it difficult to engage in goal-oriented behaviour while experiencing anxiety.

\section{Emotion regulation as predictor}

Novel theoretical frameworks emphasise that difficulties in emotion regulation are associated with the development and maintenance of anxiety disorders. ${ }^{19} \quad 26 \quad 27$ Additionally, emotion regulation skills may be required to engage in and benefit from CBT. ${ }^{23}{ }^{25}$ Furthermore, in an attempt to increase effectiveness, several newer treatments have included emotion regulation training in their treatment protocols. ${ }^{23} 2829$

The present study aims to examine attentional control and three facets of emotion regulation: (1) awareness and understanding of emotions, (2) acceptance of emotions and (3) the ability to engage in goal-directed behaviour, and refrain from impulsive behaviour when experiencing negative emotions as general predictors of outcome in CBT for anxiety disorders in a community-based mental health setting. Our secondary aim is to examine atheoretical variables (ie, sociodemographic data and clinical characteristics, such as comorbidity) as predictors of CBT outcome, as these have yet to be examined as predictors in real-world clinical settings in a mixed anxious sample.

We expect that the low ability to control attention will be associated with poorer treatment outcome. Similarly, it is expected that high scores on emotion dysregulation will be related to poorer treatment outcome. Given the exploratory nature of this study, we will also be interested in testing non-linear relationships. ${ }^{16}$

\section{METHOD}

In order to maximise external validity, we are conducting the study within two real-world treatment clinics and employing minimal exclusion criteria recruiting a sample with mixed principal anxiety disorders.

\section{Participants}

Patients ( $\mathrm{n}=112)$ with anxiety disorders (social anxiety, generalised anxiety disorder, panic disorder and agoraphobia) referred for treatment from general practitioners, hospital units or private psychiatrists to two outpatient clinics of the Mental Health System in the Capital Region of Copenhagen will be included. All patients referred to the psychiatric clinics will have a full psychiatric examination by a psychiatrist who makes the preliminary psychiatric diagnoses in accordance to International Classification of Diseases, 10th Revision (ICD-10).$^{30}$ Eligible patients are then interviewed by either a psychiatrist or a trained psychologist using the anxiety part of the MINI-International Neuropsychiatric Interview $^{31}$ to assess whether the patient fulfils the criteria for an anxiety disorder according to the Diagnostic 
and Statistical Manual of Mental Disorders (5th edition).$^{32}$ The MINI is a brief, structured diagnostic interview with excellent validity and interrater reliability. ${ }^{31}$

Patients will be eligible for the study if they (1) meet the diagnostic criteria for an anxiety disorder (social anxiety, generalised anxiety, panic disorder and agoraphobia) as primary diagnosis whether first episode or recurrent, (2) are between 18 and 60 years of age, (3) provide written informed consent and (4) speak and understand Danish. Exclusion criteria include: (1) cognitive disabilities (assessed with subscales from Wechsler Adult Intelligence Scale, fourth edition, <70), (2) alcohol or substance abuse (assessed during the preliminary consultations) or (3) receiving any other psychotherapy during the study. Patients receiving medical treatment (eg, antidepressants, benzodiazepines) will also be included in the study and the treatment will be monitored closely.

\section{Intervention}

The participants will receive 12-16 sessions of manualised group-based CBT with a maximum of eight participants in each group. The therapists are trained psychiatrists, psychologists or nurses, under supervision. The treatment manuals are developed by mental health professionals in the Capital Region of Denmark. The treatment programme includes traditional CBT components (eg, psychoeducation, identification of thoughts and feelings, exposure, cognitive restructuring and relapse prevention).

\section{Measures}

The participants will be assessed with the following measures at pretreatment and post-treatment and at 6-month follow-up.

\section{Primary outcome measure}

Beck Anxiety Inventory (BAI): ${ }^{33}$ As this study includes patients with mixed anxiety disorders, an outcome measure that measures general anxiety symptoms has been chosen. BAI is a widely used questionnaire and has 21 items measuring the intensity of general anxiety symptoms. BAI has shown high internal consistency $(\alpha=0.92)$ and high test-retest reliability, $r(81)=0.75 .^{33}$

\section{Secondary outcome measure}

Symptom checklist 90-revised (SCL-90-R): ${ }^{34}$ To measure general symptoms, the SCL-90-R will be used. The SCL-90 is a self-administered questionnaire measuring symptoms on one global scale and nine subscales: somatisation, obsessive-compulsive symptoms, interpersonal sensitivity, depression, anxiety, hostility, phobic anxiety, paranoid ideation and psychoticism. The scale has good test-retest reliability, internal consistency and construct validity. ${ }^{34}$

\section{Potential predictors}

Difficulties in Emotion Regulation Questionnaire (DERS): ${ }^{35}$ DERS is a 36-item scale designed to assess to which extent difficult emotions are impacting daily life. The DERS provides an overall score of difficulties in emotion regulation and taps six specific factors related to emotion dysregulation: (1) non-acceptance. nonacceptance of emotional responses, (2) goals: difficulty engaging in goal-oriented behaviours, (3) impulse. difficulty controlling impulses, (4) awareness: lack of emotional awareness, (5) strategies: lack of access to emotion regulation strategies and (6) clarity: lack of emotional clarity. The scale has high internal consistency $(\alpha=0.93){ }^{35}$

Attention Control Scale (ACS):20 36 The ACS is a 20-item self-report scale designed to measure two major components of attention: focusing and shifting of attention. Specifically, the ACS measures the experienced ability to focus and shift attention voluntarily. The ACS is widely used to assess executive functions and has high internal consistency $(\alpha=0.88){ }^{36}$

Theory of Visual Attention (Emotional CombiTVA paradigm) (TVA): ${ }^{37}$ This study will use TVA $^{38}$ as a framework for investigating attentional functions and attentional bias in the participants. TVA is a formal computational theory that describes attention as a set of processes which allow us to select the information that is currently relevant for behaviour. The specific paradigm used in this study, the CombiTVA, ${ }^{39}$ taps several attentional core functions such as how much material can be stored in visual shortterm memory (VSTM) and how fast visual information can be processed. Furthermore, it is possible to measure the threshold of conscious perception and the selectivity of attention, as well as to characterise how participants distribute their attention over space. TVA-based assessment of attention has primarily been conducted using simple, non-emotional stimuli (eg, letters, digits, words or simple figures), however, in this project, we will introduce the use of emotional stimuli in the CombiTVA paradigm (for a preliminary TVA-based study employing stylised emotional faces ${ }^{37}$ ). This will enable us to estimate whether, for example, negative emotional stimuli are attended preferentially or avoided, processed faster or slower, and if they affect the capacity of visual shortterm memory differently compared to neutral stimuli.

\section{Sample size}

To calculate the sample size and power, we used G*Power 3.1. First, sample size was calculated concerning the primary outcome, BAI. Previous studies have indicated reductions in BAI score after CBT treatment corresponding to effect sizes (Cohen's d) between 0.44 and $0.87 .^{40}{ }^{41}$ For choosing a conservative estimate of 0.44 , we need a sample of 57 patients to power the study sufficiently (90\%) at an $\alpha$-level of $5 \%$. Second, we calculated the required sample size for one of the predictors, DERS. A total of 84 patients is needed to obtain a power of $90 \%$ and detect a $5 \%$ increase in the explained 
variance in post BAI score when pre DERS score is included as an additional predictor in a regression model with pre BAI score and pre DERS score as predictors at a two-tailed significance level of $5 \%$ and assuming a BAI test-retest effect of $\mathrm{r}=0.75 .{ }^{33}$ Previous predictor studies in the field of anxiety disorders ${ }^{13} 1642$ have indicated a dropout rate of approximately $25 \%$ from baseline to follow-up. Hence, we aim to recruit 112 participants to make up for the expected loss of participants.

\section{Data analysis}

Our overall approach to data analysis will be linear mixed model regression analyses, in which the clustering of patients will be accounted for by inclusion of therapist-specific and group-specific random intercepts, if necessary, as judged based on levels of between-group variance in the outcome and model fit. Patients will be considered treatment completers if they have attended at least nine therapy sessions. In order to investigate if there is a difference between treatment completers and dropouts, baseline measures of BAI, SCL 90 R, DERS, ACS and CombiTVA variables will be used as predictors of treatment completion versus dropout in a logistic regression analysis.

The primary outcome measure (BAI) is measured at three time points: baseline, post-treatment and 6-month follow-up, rather than as more continuous measures during treatment. This makes the fitting of growth curves inappropriate, and similar to recent studies ${ }^{16}$ we will therefore employ a mixed-effects, analysis of covariance (ANCOVA)-like design. Baseline BAI will be included as a between-subjects covariate, and the two post-treatment assessments will be treated as two levels of the repeated-measures independent variable (TIME: post-treatment vs follow-up). The repeated measures covariance structure will be chosen based on optimal model fit. Missingness is only expected on the outcome variable, and this analytical strategy will allow us to include all patients with at least one post-treatment measurement of the outcome. To examine the main objective of this study: how attentional control and emotion regulation affect anxiety levels post-treatment, we will include the potential predictor variables as measured at baseline as covariates, both as main effects and in interaction with the TIME variable. A significant main effect of the predictor variable will indicate that the variable predicts post-treatment BAI while taking baseline BAI levels into account. A significant interaction between the predictor variable and TIME will indicate that this effect differs for the post-treatment and follow-up measurements. To explore non-linear relationships, we will also add the quadratic effects of predictor variables in a second analysis step. The predictor variables will be tested in separate models, both for the main outcome variable (BAI) and for the secondary outcome variable (SCL $90 \mathrm{R}$ ).

\section{DISSEMINATION}

Personal data about the respondents are thus protected according to cf. section 50(1)(i) of the Danish Act on Processing of Personal Data. Moreover, the Danish Committee System on Health Research Ethics was informed of the project. The committee found that the project did not need separate ethical approval, since it does not include any biomedical intervention. Study findings will be disseminated through peer-reviewed publications and conference presentations.

Contributors SKKN and SV have primarily designed the study. SIFD and KBW-T provided statistical advice. IH provided research design advice. SKKN wrote the manuscript. SV, SIFD, IH and KBW-T proofed and edited the manuscript.

Funding The work is supported by Kristian Holt Hansen Foundation and Aase \& Ejnar Danielsen Foundation.

\section{Competing interests None declared}

Ethics approval The study is approved by the Ethical Board, Department of Psychology, University of Copenhagen and by the Danish Data Protection Agency (OK308627).

Provenance and peer review Not commissioned; externally peer reviewed.

Open Access This is an Open Access article distributed in accordance with the Creative Commons Attribution Non Commercial (CC BY-NC 4.0) license, which permits others to distribute, remix, adapt, build upon this work noncommercially, and license their derivative works on different terms, provided the original work is properly cited and the use is non-commercial. See: http:// creativecommons.org/licenses/by-nc/4.0/

\section{REFERENCES}

1. Kessler RC, Petukhova M, Sampson NA, et al. Twelve-month and lifetime prevalence and lifetime morbid risk of anxiety and mood disorders in the United States: anxiety and mood disorders in the United States. Int J Methods Psychiatr Res 2012;21:169-84.

2. Lépine J-P. The epidemiology of anxiety disorders: prevalence and societal costs. J Clin Psychiatry 2002;63(Suppl 14):4-8.

3. Greenberg PE, Sisitsky T, Kessler RC, et al. The economic burden of anxiety disorders in the 1990s. J Clin Psychiatry 1999;60:427-35.

4. Stewart RE, Chambless DL. Cognitive-behavioral therapy for adult anxiety disorders in clinical practice: a meta-analysis of effectiveness studies. J Consult Clin Psychol 2009;77:595-606.

5. van Apeldoorn FJ, Stant AD, van Hout WJPJ, et al. Cost-effectiveness of CBT, SSRI, and CBT+SSRI in the treatment for panic disorder. Acta Psychiatr Scand 2014;129:286-95.

6. Loerinc AG, Meuret AE, Twohig MP, et al. Response rates for CBT for anxiety disorders: need for standardized criteria. Clin Psychol Rev 2015;42:72-82.

7. Persons JB, Silberschatz $\mathrm{G}$. Are results of randomized controlled trials useful to psychotherapists? J Consult Clin Psychol 1998;66:126-35.

8. Howard KI, Moras K, Brill PL, et al. Evaluation of psychotherapy: efficacy, effectiveness, and patient progress. Am Psychol 1996;51:1059-64

9. Seligman ME. The effectiveness of psychotherapy. The Consumer Reports study. Am Psychol 1995;50:965-74.

10. Holmqvist R, Philips B, Barkham M. Developing practice-based evidence: benefits, challenges, and tensions. Psychother Res J Soc Psychother Res 2014;25:20-31.

11. Taylor S, Abramowitz JS, McKay D. Non-adherence and non-response in the treatment of anxiety disorders. J Anxiety Disord 2012;26:583-9.

12. Olatunji BO, Cisler JM, Tolin DF. A meta-analysis of the influence of comorbidity on treatment outcome in the anxiety disorders. Clin Psychol Rev 2010;30:642-54.

13. Chambless DL, Renneberg B, Gracely EJ, et al. Axis I and II comorbidity in agoraphobia: prediction of psychotherapy outcome in a clinical setting. Psychother Res 2000;10:279-95.

14. Porter E, Chambless DL. A systematic review of predictors and moderators of improvement in cognitive-behavioral therapy for panic disorder and agoraphobia. Clin Psychol Rev 2015;42:179-92. 
15. Schneider RL, Arch JJ, Wolitzky-Taylor KB. The state of personalized treatment for anxiety disorders: a systematic review of treatment moderators. Clin Psychol Rev 2015;38:39-54.

16. Wolitzky-Taylor KB, Arch JJ, Rosenfield D, et al. Moderators and non-specific predictors of treatment outcome for anxiety disorders: a comparison of cognitive behavioral therapy to acceptance and commitment therapy. J Consult Clin Psychol 2012;80:786-99.

17. Niles AN, Mesri B, Burklund LJ, et al. Attentional bias and emotional reactivity as predictors and moderators of behavioral treatment for social phobia. Behav Res Ther 2013;51:669-79.

18. Bar-Haim Y, Lamy D, Pergamin L, et al. Threat-related attentional bias in anxious and nonanxious individuals: a meta-analytic study. Psychol Bull 2007;133:1-24.

19. Campbell-Sills L, Barlow DH. Incorporating Emotion Regulation into Conceptualizations and Treatments of Anxiety and Mood Disorders. I: Handbook of emotion regulation. New York, NY, USA: Guilford Press, 2007:542-59.

20. Derryberry D, Reed MA. Anxiety-related attentional biases and their regulation by attentional control. $J$ Abnorm Psychol 2002;111:225-36.

21. Eysenck MW, Derakshan N, Santos R, et al. Anxiety and cognitive performance: attentional control theory. Emotion 2007;7:336-53.

22. Mennin DS, McLaughlin KA, Flanagan TJ. Emotion regulation deficits in generalized anxiety disorder, social anxiety disorder, and their co-occurrence. J Anxiety Disord 2009;23:866-71.

23. Moses EB, Barlow DH. A new unified treatment approach for emotional disorders based on emotion science. Curr Dir Psychol Sci 2006;15:146-50.

24. Beck AT, Emery G, Greenberg RL. Anxiety disorders and phobias, a cognitive perspective. Basic Books, 1985.

25. Craske MG, Kircanski K, Zelikowsky M, et al. Optimizing inhibitory learning during exposure therapy. Behav Res Ther 2008;46:5-27.

26. Mennin DS, Heimberg RG, Turk CL, et al. Preliminary evidence for an emotion dysregulation model of generalized anxiety disorder. Behav Res Ther 2005;43:1281-310.

27. Simons JS, Gaher RM. The distress tolerance scale: development and validation of a self-report measure. Motiv Emot 2005;29:83-102.

28. Cloitre M, Koenen KC, Cohen LR, et al. Skills training in affective and interpersonal regulation followed by exposure: a phase-based treatment for PTSD related to childhood abuse. J Consult Clin Psychol 2002;70:1067-74.
29. Mennin DS, Heimberg RG, Turk CL, et al. Applying an emotion regulation framework to integrative approaches to generalized anxiety disorder. Clin Psychol Sci Pract 2002;9:85-90.

30. WHO. I International Classification of Diseases (ICD) WHO

31. Sheehan DV, Lecrubier Y, Sheehan $\mathrm{KH}$, et al. The Mini-International Neuropsychiatric Interview (M.I.N.I): the development and validation of Structured Diagnostic Psychiatric Interview for DSM-IV and ICD-10. J Clin Psychiatry 1998;59(Suppl 20):22-33;quiz 34-57.

32. American Psychiatric Association. Diagnostic and Statistical Manual of Mental Disorders [Internet]. American Psychiatric Association, 2013.

33. Beck AT, Epstein N, Brown G, et al. An inventory for measuring clinical anxiety: psychometric properties. J Consult Clin Psychol 1988;56:893-7.

34. Derogatis LR. SCL-R-90: Symptom Checklist-90-R (SCL-90-R) Administration, Scoring, and Procedures Manual. Minneapolis: National Computer Systems, 1994.

35. Gratz KL, Roemer L. Multidimensional assessment of emotion regulation and dysregulation: development, factor structure, and initial validation of the difficulties in emotion regulation scale. J Psychopathol Behav Assess 2004;26:41-54

36. Derryberry D, Reed MA. 13 A Multidisciplinary Perspective on Attentional Control. Attract Distraction Action Mult Perspect Attentional Capture 2001:133:325.

37. Barratt D, Bundesen C. Attentional capture by emotional faces is contingent on attentional control settings. Cogn Emot 2012;26:1223-37.

38. Bundesen C. A theory of visual attention. Psychol Rev 1990;97:523-47.

39. Vangkilde S, Bundesen C, Coull JT. Prompt but inefficient: nicotine differentially modulates discrete components of attention. Psychopharmacology 2011;218:667-80.

40. Dugas MJ, Ladouceur R, Léger E, et al. Group cognitive-behavioral therapy for generalized anxiety disorder: treatment outcome and long-term follow-up. J Consult Clin Psychol 2003;71:821-5.

41. Schuurmans J. A randomized, controlled trial of the effectiveness of cognitive-behavioral therapy and sertraline versus a waitlist control group for anxiety disorders in older adults. Am J Geriatr Psychiatry 2006;14:255-63.

42. Westra HA, Dozois DJA, Marcus M. Expectancy, homework compliance, and initial change in cognitive-behavioral therapy for anxiety. J Consult Clin Psychol 2007;75:363-73. 